\title{
LA EDUCACIÓN ALIMENTARIA DE LOS NIÑOS
}

\author{
Sabina Russo \\ Doctorado en Educación en la Universidad de Valencia (España), \\ sabyrusso@alice.it
}

Recepción Artículo: 26 febrero 2020

Admisión Evaluación: 4 marzo 2020

Informe Evaluador 1: 13 marzo 2020

Informe Evaluador 2: 15 Marzo 2020

Aprobación Publicación: 20 abril 2020

\section{SUMMARY}

Over the years, there has been a progressive increase in the number of overweight and obese people of all ages and social realities in Europe and around the world, making obesity one of the most common public health problems pressing. Obesity is a problem that does not forgive even the smallest children. The latest published data shows that children in southern European countries are those with the greatest weight problems (between $18 \%$ and $21 \%$ ); in Italy one in five children is obese. These data led to an investigation in Ribera, a country in Sicily (Italy), aimed at promoting the improvement of children's nutrition by raising awareness about the bad eating habits related to eating breakfast and snacks at school. Specifically, the research, based on the idea that teachers and parents should jointly help children develop and promote knowledge and awareness of healthy eating habits, wanted to demonstrate that children's use of a newspaper weekly snacks at school contributes to the improvement of eating habits. Keywords: nutrition, overweight, obesity, children, family and school

\section{RESUMEN}

A lo largo de los años, se ha producido un aumento progresivo del número de personas con sobrepeso y obesas de todas las edades y realidades sociales en Europa y en todo el mundo, lo que hace de la obesidad uno de los problemas de salud pública más acuciantes. La obesidad es un problema que no perdona ni siquiera a los niños más pequeños.

Los últimos datos publicados muestran que los niños de los países del sur de Europa son los que tienen mayores problemas de peso (entre el $18 \%$ y el $21 \%$ ); en Italia uno de cada cinco niños es obeso. Esos datos dieron lugar a una investigación en Ribera, un país de Sicilia (Italia), destinada a promover la mejora de la nutrición infantil mediante la sensibilización sobre los malos hábitos alimentarios relacionados con el consumo de desayunos y refrigerios en la escuela. Concretamente, la investigación, partiendo de la idea de que los maestros y los padres deben ayudar conjuntamente a los niños a desarrollar el conocimiento y la conciencia de los hábitos alimentarios sanos y a promoverlos, quería demostrar que el uso por parte de los niños de un diario semanal de aperitivos en la escuela contribuye a la mejora de los hábitos alimentarios.

Palabras clave: nutrición, sobrepeso, obesidad, niños, familia y escuela 


\section{LA EDUCACIÓN ALIMENTARIA DE LOS NIÑOS}

\section{SOMMARIO}

Nel corso degli anni, c'è stato un progressivo aumento del numero di persone in sovrappeso e obese di tutte le età e realtà sociali in Europa e nel mondo, rendendo l'obesità uno dei problemi di salute pubblica più comuni pressatura. L'obesità è un problema che non perdona nemmeno i bambini più piccoli. Gli ultimi dati pubblicati mostrano che i bambini nei paesi dell'Europa meridionale sono quelli con i maggiori problemi di peso (tra il 18\% e il 21\%); in Italia un bambino su cinque è obeso. Questi dati hanno portato a un'indagine a Ribera, un paese in Sicilia (Italia), volto a promuovere il miglioramento della nutrizione dei bambini aumentando la consapevolezza delle cattive abitudini alimentari legate al fare colazione e agli spuntini a scuola. In particolare, la ricerca, basata sull'idea che insegnanti e genitori dovrebbero aiutare insieme i bambini a sviluppare e promuovere la conoscenza e la consapevolezza delle sane abitudini alimentari, ha voluto dimostrare che i bambini usano un giornale gli spuntini settimanali a scuola contribuiscono al miglioramento delle abitudini alimentari.

Parole chiave: alimentazione, sovrappeso, obesità, bambini, famiglia e scuola

\section{LA EDUCACIÓN ALIMENTARIA DE LOS NIÑOS: LA OBESIDAD.}

\section{Introducción.}

En los últimos decenios se ha observado un aumento gradual del número de personas con sobrepeso y obesas de todos los grupos de edad en Europa y en todo el mundo y, como consecuencia, uno de los problemas de salud pública más acuciantes con importantes costos sociales.

Uno de los problemas es el de la obesidad, que no conoce fronteras ni hace distinciones entre las clases sociales, en el sentido de que si antes se consideraba erróneamente como la enfermedad de las clases sociales ricas, hoy en día se ha demostrado ampliamente que esto no es cierto. Hoy en día es posible afirmar que, de hecho, hay porcentajes cada vez más elevados de personas que sufren de obesidad en las clases sociales desfavorecidas y en los países desfavorecidos con problemas de malnutrición (Fabrizio, 2011).

La obesidad no sólo es un problema para los adultos, sino también para los niños, y está influenciada por factores culturales, ambientales, económicos, psicológicos y genéticos, así como, por supuesto, por la actividad física y la dieta, aunque ésta última sea menos importante y decisiva de lo que pensamos ( $\mathrm{Ng}$, Fleming, Robinson, Thomson, Graetz \& Margono, 2013).

\section{Marco teórico: Definición y estudios.}

Por lo tanto, es necesario definir la obesidad eliminando ciertas "creencias" y analizando las diversas implicaciones sociales y sanitarias. En primer lugar, cabe señalar que la obesidad es el aumento de la masa corporal distribuida en determinadas partes del cuerpo, al que se vincula el aumento de enfermedades como la diabetes mellitus de tipo 2, las enfermedades cardiovasculares y la hipertensión, y la mortalidad. (Flegal, Graubard, Williamson \& Gail, 2007). El Overseas Development Institute también ha demostrado que la obesidad, a diferencia de lo que comúnmente se piensa, no es una enfermedad de los países ricos y, de hecho, el número de personas que sufren de sobrepeso y obesidad es mayor en los países en desarrollo que en los países ricos (904 millones de personas en los países en desarrollo y 557 millones en el resto del mundo) y las tasas de obesidad siguen aumentando entre las personas de baja condición socioeconómica y entre los grupos étnicos minoritarios (OMS, 2018); La OMS ha determinado que el número de niños con sobrepeso u obesidad en África ha aumentado en un 50\% aproximadamente desde 2000. También se determinó que alrededor de la mitad de los niños menores de 5 años con sobrepeso u obesidad vivían en Asia en 2016 (OMS, 2019).

Otro estudio interesante que refuta uno de los clichés sobre la obesidad es el de Ritchie y Roser (Ritchie \& Rose, 2019) que demuestra la clara correlación entre la prosperidad y la obesidad y que el número de personas obesas, adultos y niños, ha aumentado a lo largo de los años en todo el mundo.

Todos los estudiosos coinciden ahora en que la obesidad es una enfermedad crónica que puede afectar a la salud de niños y adultos de muchas maneras: salud inmediata, educación, rendimiento y calidad de vida. 
Actualmente se ha establecido que los niños obesos tienen muchas probabilidades de ser obesos en la edad adulta y corren el riesgo de desarrollar enfermedades crónicas no transmisibles graves. Si no se invierte la tendencia contemporánea, para 2030 la mitad de la población mundial será obesa y ello supondrá una carga (en términos de pérdida de productividad, coste de los servicios e inversiones para invertir la tendencia) del $28 \%$ del PIB (Producto Interior Bruto) mundial.

A pesar de los numerosos estudios realizados hasta la fecha, todavía no existe una opinión compartida en la comunidad científica sobre los factores que determinan la obesidad. Según algunas personas, la obesidad se debe a una dieta excesiva, especialmente si la cantidad de calorías ingeridas supera la cantidad necesaria para las necesidades energéticas, y a una mala calidad de los alimentos consumidos (alimentos con menos nutrientes y más calorías); por lo tanto, existe una sobrealimentación relativa, es decir, una dieta que altera la sensación de hambre en las personas obesas en comparación con las que tienen un peso normal (Gremigni \& Letizia, 2011). Por otra parte, otros estudios han abordado la relación entre la obesidad y el nivel de escolaridad y otros índices siempre vinculados al nivel cultural y socioeconómico de los individuos. Tampoco faltaron estudios médicos que se centraran en el patrimonio genético. Como no ha sido posible correlacionar las causas con un gen, durante años se ha hablado de la "teoría del gen ahorrativo", según la cual algunos genes han evolucionado de tal manera que favorecen la obesidad para permitir que la humanidad pase indemne entre la hambruna y los tiempos difíciles. Sin embargo, nuevas pruebas científicas indican que ninguno de los genes implicados en la predisposición a la obesidad (hay 115) ha desempeñado nunca esta función. Interesante a este respecto es el estudio de Wang y Speakman (2016).

Otra contribución interesante al estudio de la obesidad fue el estudio de la leptina. La leptina es una hormona producida por el tejido adiposo que tiene la tarea de regular la ingesta de alimentos a través de la comunicación con el sistema nervioso central.

Pero la obesidad también está influenciada por la familia, la escuela y el ambiente y estilo de vida amigables (Rotella, 1980; Faliva, 2011). El estilo de vida es ciertamente uno de los factores que determinan la obesidad y se han realizado muchos estudios e investigaciones para determinar el peso que este factor tiene en la incidencia de este fenómeno (Hobbs, Pearson, Foster \& Biddle, 2015). Otro aspecto importante que afecta a la obesidad infantil es la actividad física; cada vez son más los niños que no se mueven y son sedentarios, prefieren los videojuegos a los juegos de movimiento al aire libre y esto también sucede porque las oportunidades de moverse y hacer ejercicio son cada vez más reducidas.

\section{Implicación y significación educativa:}

Hablando de la obesidad, el elemento más alarmante es que cada vez más niños en la infancia la padecen (OMS, 2018); de la familia derivan los hábitos alimentarios de los niños y es una fuente de calor, sustento y afecto para el niño pero, innegablemente, también de frustración y estrés.

La escuela y la familia, como todo el mundo sabe ya, son los principales organismos educativos. Ambos, de hecho, tienen como propósito la educación del niño. La educación informal es responsabilidad de la familia, mientras que la educación formal es responsabilidad de la escuela. Las funciones no están claramente separadas, por lo que la relación entre ellas debe ser de colaboración, diálogo y respeto mutuo. A través de la relación escuela-familia, Ios alumnos pueden adquirir, más allá del contenido y las habilidades, los valores necesarios para desarrollar la responsabilidad, la colaboración y la gestión de conflictos.

La Organización Mundial de la Salud, con el establecimiento de la Iniciativa Europea de Vigilancia de la Obesidad Infantil (COSI) de la OMS, ha creado un sistema de vigilancia de la obesidad infantil en Europa e Italia participa en este sistema con el programa de vigilancia "OKkio alla salute"1 con el que recoge datos sobre nuestro país. El objetivo principal del programa es realizar una encuesta constante y disponer de informes cada dos años que permitan tener datos suficientes y constantes sobre la muestra a lo largo de los años para poder, a través de su lectura, conocer y comprender los avances en la lucha contra la obesidad en la infancia. El programa de vigilancia "OKkio to Health" ha demostrado que una política de escucha, información y vigilancia es la forma correcta 


\section{LA EDUCACIÓN ALIMENTARIA DE LOS NIÑOS}

de luchar contra esta enfermedad y permitir a las personas buscar su propio bienestar y adquirir un estilo de vida saludable desde la infancia, que luego se convertirá en una educación alimentaria de por vida. Sólo así se pueden limitar los daños y los costos de la epidemia de obesidad, tanto en lo social como en lo económico.

\section{ESTUDIO EMPÍRICO}

La investigación, utilizando una metodología cualitativa-cuantitativa, se ha fijado el objetivo general de promover una mejora de la nutrición infantil mediante la concienciación de los malos hábitos alimentarios relacionados con el consumo de desayunos y meriendas en la escuela. El estudio fue también una investigación-intervención, en la que se desarrollaron diferentes fases, en una secuencia lógica y compleja, que dio lugar a un proceso en el que la recopilación de datos se entrelazaba con la promoción del cambio; un estudio con objetivos de investigación y cambio.

Si el propósito general se refiere a los hábitos alimentarios más o menos saludables de los niños sicilianos, los objetivos específicos se refieren tanto a la recopilación de datos (investigación) como a la promoción y mejora del comportamiento alimentario (intervención), con el fin de demostrar la eficacia de la intervención realizada.

En lo que respecta a la parte de investigación, los objetivos específicos eran investigar los hábitos alimentarios relacionados con el consumo de desayuno y refrigerios en la escuela; identificar los principales errores nutricionales en la edad escolar.

En cuanto a los objetivos específicos de la intervención implementada (uso del diario de la merienda semanal, el desayuno en el aula y la merienda de frutas), los objetivos fueron: adquirir hábitos de desayuno; tener una merienda adecuada en la escuela; concienciar a las familias de la importancia de una alimentación saludable.

Se esperaba que, tras la aplicación del diario semanal de refrigerios en la escuela, así como de las dos iniciativas "desayuno en la clase" y "refrigerio con fruta", los hábitos alimentarios de los niños mejorarían.

\section{Participantes}

Como optaron por realizar una intervención de búsqueda, no se realizó ningún muestreo aleatorio; los participantes se eligieron de forma voluntaria.

En la investigación, realizada durante el año escolar 2017/2018, 153 alumnos asistieron a la primera clase de la escuela primaria de los Institutos Integrales "Don Bosco", "Imbornone" y "E. Cufalo" en Ribera (AG), Sicilia, y sus padres.

Concretamente, los participantes tienen entre 5 años y 10 meses y 7 años de edad, con una edad media de unos 6 años y 3 meses y una desviación estándar de unos 4 meses, de los cuales el 55,56\% son mujeres y el 44,44\% hombres. Todos sus padres (306 personas) también participaron en la investigación, incluidas 10 madres de nacionalidad extranjera.

Los padres casi todos tienen un diploma de secundaria (93 padres y 97 madres), mientras que alrededor del $20 \%$ tienen un título (26 madres y 34 madres), el diploma de secundaria lo tienen 31 padres y 20 madres, y finalmente sólo 3 padres y 2 madres tienen sólo un diploma de primaria.

\section{Instrumentos utilizados}

A fin de obtener resultados fiables y comparables con los del contexto nacional italiano, se decidió utilizar el cuestionario dirigido a los alumnos y el cuestionario dirigido a los padres ya utilizado en el contexto nacional por "OKkio alla salute".

Concretamente, el cuestionario dirigido a los alumnos investiga un período limitado de tiempo desde la tarde del día anterior hasta la mañana de la encuesta y se compone de 15 preguntas, 13 de las cuales son de respuesta cerrada y 2 de respuesta abierta, que investigan los hábitos alimentarios en el desayuno y la merienda en la escuela, la actividad motriz y el comportamiento sedentario, como el uso de los videojuegos y la televisión, mientras que el cuestionario dirigido a los padres consta de 15 preguntas de respuesta cerrada, divididas en 5 áreas: el primero sobre las actividades que el niño realiza después de la escuela (actividad física, videojuegos, televi- 
sión); el segundo sobre la alimentación del niño (en particular, sobre el consumo de fruta y verdura y de bebidas azucaradas y/o gaseosas); el tercero sobre la percepción del estado de peso y la actividad motriz de sus hijos; el cuarto sobre algunos datos sociodemográficos de los padres; el quinto sobre los datos antropométricos de los niños (edad, sexo, peso, altura).

El cuestionario dirigido a los alumnos se administró en el aula, individualmente y por escrito al principio y después de la aplicación del diario semanal de aperitivos.

Las preguntas dirigidas a los padres abarcaban los mismos temas, pero durante un período de tiempo más largo.

A partir de los datos proporcionados por los padres sobre el peso expresado en kilogramos y el cuadrado de la altura expresado en metros, se calculó el Índice de Masa Corporal (IMC) y, para estimar la condición de sobrepeso y el riesgo de obesidad en los niños, se decidió utilizar el IMC porque la literatura muestra que el IMC es un buen indicador del estado de peso incluso en la infancia. Por último, siguiendo las indicaciones del Instituto Nacional de Investigación para la Alimentación y la Nutrición (INRAN), sobre la base de lo declarado por los niños, se procedió a evaluar el desayuno y el desayuno como comidas adecuadas o no adecuadas.

Los profesores, durante las reuniones que les fueron dedicadas, proporcionaron información importante sobre el horario escolar (tiempo completo, semana corta, etc.), la educación motriz (cuántas veces a la semana, durante cuánto tiempo, cómo se lleva a cabo), el tipo de bocadillo que los niños trajeron al aula y las bebidas, para ver si estaban comiendo una dieta saludable 0 trayendo bocadillos no saludables.

En esta fase, con el fin de comprender mejor los hábitos alimentarios de los niños durante el tiempo de juego, se hicieron observaciones de los participantes de las que se desprendió que los niños no consumen alimentos saludables durante el tiempo de juego, con la excepción de algunos alumnos que comieron yogur, algunos que comieron fruta y otros que comieron un sándwich relleno de verduras y/o ensalada.

También en esta fase preliminar se celebró una reunión informativa con las familias de las clases involucradas para discutir las actividades que se iban a llevar a cabo durante el año escolar y para ilustrar ya las propuestas de mejora en relación con la merienda en la escuela y el inicio del diario semanal de la merienda. Durante la reunión, a la que también asistió un biólogo nutricionista, se destacó también la importancia del desayuno, la fruta y las verduras, con el objetivo de orientar a los niños hacia una elección de alimentos más saludables, sobre todo educándolos en una dieta sana y correcta, y se hizo hincapié en la colaboración de las familias para continuar la labor realizada por los profesores.

Los cuestionarios se administraron a los alumnos (noviembre) y a sus padres (diciembre).

Sobre la base de la situación que surgió de la encuesta cognoscitiva en relación con la alimentación y los refrigerios en la escuela, es decir, que los niños no consumen un refrigerio adecuado en la escuela, se propuso un diario semanal de refrigerios para el resto del año escolar; esta fue la fase de la intervención que tuvo lugar de enero a mayo. Esta fase se asoció, la segunda semana de cada mes, a la actividad de vigilancia relativa con el uso de la red de observación, para detectar cuántos niños seguían el diario de alimentos respetando la merienda del día.

Además, se implementaron el desayuno en el aula y el refrigerio de frutas.

Por último, en junio, a fin de evaluar las posibles mejoras y cambios, se entregó a los alumnos el mismo cuestionario utilizado en la fase inicial,

LOS datos reunidos se incluyeron en el programa de análisis estadístico SPSS y se sometieron a un análisis descriptivo y a pruebas estadísticas que permiten verificar la hipótesis inicial comparando los datos antes y después de la intervención.

Los análisis de datos fueron: análisis de frecuencias y frecuencias porcentuales, cálculo de promedios y desviaciones estándar, análisis de regresiones, comparación entre los datos recogidos antes y después de la intervención (chi cuadrado). 


\section{LA EDUCACIÓN ALIMENTARIA DE LOS NIÑOS}

\section{El estado ponderal de los niños}

El primer dato que se ha investigado es el estado de peso, y se descubrió que el $40 \%$ de los niños que participaron en la investigación tienen sobrepeso y entre ellos alrededor del 20\% ya padecen la condición de obesidad; una cifra alarmante que, sin embargo, refleja los promedios regionales, ya que en Sicilia el problema de la obesidad y el sobrepeso en los niños está más extendido que en el resto de Italia. Sólo poco más de la mitad de los niños tendrían un peso ideal y un pequeño porcentaje (5,23\%) están por debajo del peso ideal. Entre los niños con sobrepeso hay una distribución uniforme entre los sexos, mientras que entre los niños con bajo peso hay mujeres.

En la actualidad se confirma en la literatura que los padres no siempre reconocen un exceso de peso en su hijo (Huang, Becerra, Oda, Walker, Xu, Donohue, Chen, Curbelo \& Breslow, 2007), especialmente si pertenecen a un nivel socioeconómico más bajo (Rhee, De Lago, Arscott-Mills, Mehta \& Davis, 2005).

Admitir que el hijo tiene sobrepeso o es obeso ya sería el primer paso en la lucha contra la obesidad infantil (He \& Evans, 2007), especialmente para la madre, que sigue desempeñando el papel principal en nuestra sociedad en la educación, la elección y la preparación de los alimentos para sus hijos y toda la familia.

En consonancia con lo que se ha informado en las investigaciones científicas nacionales, los datos reunidos han mostrado una dificultad para reconocer una condición de sobrepeso y/u obesidad en sus hijos. De hecho, se encontró que para el 80,39\% de los padres el peso de su hijo es normal, para alrededor del 12\% su hijo tiene un poco de sobrepeso, y para el 8\% restante un peso inferior al normal; nadie cree que su hijo tenga "mucho" sobrepeso. Por lo tanto, se puede observar que los padres del $49 \%$ de los niños con sobrepeso y del $10 \%$ de los niños obesos consideran que su hijo tiene un peso normal o incluso un peso inferior al normal.

Los padres tienden generalmente a subestimar el peso de sus hijos y, de hecho, sólo el $4 \%$ de los niños con peso normal se perciben como ligeramente obesos, alrededor de la mitad de los niños con sobrepeso se consideran de peso normal y sólo el 13\% perciben su verdadera obesidad.

Otro aspecto que ha surgido y que necesita atención es la percepción de los padres de la cantidad de comida que toman sus hijos. Cuando se les pregunta "Según ella, el niño come: poco, bien o demasiado", alrededor del $76 \%$ de los padres creen que sus hijos comen la cantidad adecuada, con un porcentaje más alto entre las mujeres (41,83\% frente a $34,64 \%)$; mientras que se considera que los hombres "comen" más que las mujeres.

Una comparación entre la percepción de los padres de sus hijos como de bajo peso, peso normal o sobrepeso y la evaluación de su dieta muestra que alrededor del 16\% de los padres consideran que su hijo come poco, pero sólo el 8\% considera que su hijo está por debajo de su peso. En lo que respecta a la sobrealimentación, ocurre lo contrario: un porcentaje menor cree que su hijo come demasiado (casi un $8 \%$ ) que los que (casi un $12 \%)$ creen que su hijo tiene sobrepeso.

Esto podría deberse en parte al hecho de que la dieta de los niños con sobrepeso u obesidad se ha modificado como resultado de su sobrepeso.

Sin embargo, lo más probable es que este resultado pueda interpretarse como una tendencia de los padres a comer en exceso a sus hijos porque no tienen la información adecuada sobre los riesgos de una mala nutrición. En otras palabras, parece que algunos padres creen que su hijo tiene sobrepeso cuando come lo adecuado (y no "demasiado") y que, por el contrario, su hijo tiene un peso normal cuando come poco (y no "lo adecuado"). Si se observan los porcentajes, de hecho, se observa una diferencia de 8 puntos porcentuales entre el porcentaje de niños que se considera que comen poco y el porcentaje de niños que se considera que tienen un peso inferior al normal. Además, surge una diferencia de 4 puntos porcentuales entre la cantidad de niños considerados con sobrepeso y el porcentaje de niños que se considera que comen demasiado. Esto sugiere que los padres tienden a subestimar la cantidad de alimentos en la dieta de sus hijos en comparación con la percepción del peso, que a su vez se subestima en comparación con una evaluación objetiva (como se ha señalado anteriormente).

Los resultados de la investigación confirman que, para prevenir la obesidad en los niños y concienciar a la comunidad de los riesgos del sobrepeso, los padres deben tomar medidas porque son los principales responsables de la nutrición y los hábitos alimentarios de sus hijos. 


\section{Los predictores del estado antropométrico}

La presente investigación tuvo como objetivo investigar los posibles predictores del estado antropométrico, y del análisis de las regresiones lineales surgieron dos de ellos: la cantidad de alimentos ingeridos según la percepción de los padres (demasiado, demasiado poco, la correcta) y la cantidad de horas que se pasan viendo la televisión.

Todas las demás variables detectadas por los cuestionarios no eran estadísticamente significativas. Por lo tanto, es sorprendente que el sobrepeso esté más relacionado con las actividades sedentarias (en particular, ver la televisión) que con la actividad física y/o deportiva y con todas las demás variables sociodemográficas (por ejemplo, las calificaciones educativas y el empleo de los padres, que son estadísticamente significativas en otras encuestas nacionales).

\section{La intervención}

Sobre la base de los datos recogidos en una etapa temprana, se propuso el diario semanal de refrigerios (véase la foto 1), con el objetivo de mejorar el consumo de refrigerios de los niños en la escuela y orientarlos de manera estable hacia pautas nutricionalmente equilibradas.

Foto 1 - El diario semanal de la merienda en la escuela.

\section{DIARIO SETTIMANALE DELLA MERENDA A SCUOLA}

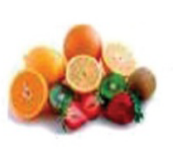

Per una sana alimentazione ricorda di fare sempre una colazione adeguata

e di mangiare a merenda 2 volte a settimana frutta e verdura.

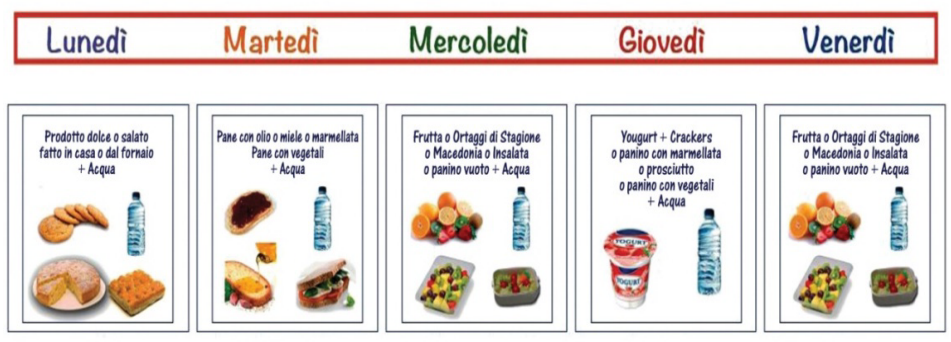

Scuola Primaria:

Classe:

El diario se creó como parte del Proyecto de Educación Alimentaria "La fruta va a la escuela" de la Oficina de Educación Sanitaria del distrito de Sciacca (AG), en colaboración con el Departamento de Agricultura y Políticas Alimentarias (SOAT de Sciacca) y la asociación cultural "Empedocle" de pediatras de Sciacca, que funcionan como un grupo de trabajo en todas las escuelas primarias de Sciacca, iniciado en 2009 y en funcionamiento en el momento de redactar el presente informe.

El diario programa el consumo de diferentes alimentos durante la semana para superar la monotonía de la comida, a menudo típica de la dieta de los niños. El diario fue observado durante cinco meses, de enero a mayo de 2018.

Además de la administración del diario cada dos semanas del mes, se realizó una observación sistemática en el aula a través de una rejilla de observación. 


\section{LA EDUCACIÓN ALIMENTARIA DE LOS NIÑOS}

La intervención incluyó, con la colaboración de las familias y los maestros, la organización de un desayuno en clase con productos no industriales pero de panadería con el objetivo de destacar la importancia de un desayuno sano y equilibrado (febrero) y la merienda en clase con fruta de temporada y la visualización de vídeos, seguida de un debate con los niños sobre la importancia y los beneficios de comer fruta y verdura en color (abril). La experiencia de desayunar en el aula, gracias al efecto de "elección de grupo", hizo que incluso los niños que no suelen desayunar antes de ir a la escuela experimentaran los beneficios del desayuno mientras que, al comer la fruta y ver el vídeo, les hizo reflexionar sobre el significado de una dieta correcta, que incluye cinco porciones de fruta y verdura, frescas en temporada y de diferentes colores.

La experiencia de la fruta en la clase hizo que incluso los niños que no suelen comerla comenzaran a comerla en lugar de los bocadillos habituales, como sándwiches con salchichas, chocolates, salchichas, bebidas azucaradas; descubrieron la bondad de la fruta. Además, incluso en el caso de los niños que suelen comer fruta, el beneficio de aumentar la confianza en sí mismos se ha logrado mediante la abolición del sentido de inferioridad que a veces puede acompañar a los niños que comen fruta como tentempié.

El objetivo de las reuniones relacionadas con el desayuno en clase y la fruta era concienciar a las familias, y no sólo a los niños, sobre la importancia del desayuno y los beneficios de la fruta.

En la fase final, se examinaron los cambios que se han producido tras la adopción de un diario semanal de aperitivos, los días de concienciación para una alimentación sana con la participación de las familias (desayuno en la escuela). Con ese fin, se dio a los niños el mismo cuestionario utilizado antes de la intervención, de manera que fue posible hacer comparaciones de las variables no paramétricas detectadas en las fases previas y posteriores a la intervención, aplicando la prueba del ji cuadrado.

Una dieta alta en calorías se asocia en los niños con un aumento de peso corporal, un aumento que tiende a mantenerse 0 incluso a seguir creciendo progresivamente durante la edad adulta (Dasgupta \& Hauspie, 2013). Por el contrario, un correcto estado nutricional está asociado a una dieta cualitativamente equilibrada, en términos de equilibrio entre grasas, proteínas y carbohidratos, y una adecuada distribución de las comidas a lo largo del día.

Por ello, muchas de las preguntas del cuestionario, tanto de los alumnos como de los padres, se referían a sus hábitos alimentarios y la intervención posterior tenía por objeto mejorar los hábitos alimentarios en beneficio, sobre todo, de un mayor consumo de fruta.

Varios estudios realizados (Gómez-Pinilla, 2008) han demostrado que un desayuno adecuado mejora la salud, así como el rendimiento cognitivo, la capacidad de aplicación y la concentración, especialmente en los niños.

En nuestro estudio, antes de la intervención, la gran mayoría de los niños tenían el hábito de desayunar por la mañana antes de ir a la escuela; el desayuno se basaba en la mayoría de los casos en leche y galletas, mientras que nadie consumía fruta, mermelada o huevos. La merienda en la escuela era consumida regularmente por todos los niños (excepto dos), pero la calidad de la comida deja mucho que desear: sólo un pequeño porcentaje come fruta, mientras que el consumo de sándwiches y bocadillos está muy extendido.

Una de las cuestiones más importantes para una correcta nutrición en la infancia está representada por la ingesta diaria de frutas y verduras, que es muy descuidada por los niños y de hecho sólo una pequeña minoría (2\%) la utiliza en todas las comidas diarias (5 o más veces al día), el $8 \%$ nunca la come, alrededor de un tercio de ellos la come de 2 a 4 veces al día y otro tercio alrededor de 1 vez al día, el 20\% restante consume frutas y verduras sólo unas pocas veces a la semana.

La acción de concienciación y prevención, Ilevada a cabo mediante la experiencia del diario semanal (que incluía dos días a la semana con fruta), el desayuno en clase junto con las familias y un refrigerio en la escuela con fruta de temporada, acompañado de la visualización de un vídeo y un debate en grupo sobre los beneficios de la fruta, han introducido algunos cambios. Por lo tanto, el proyecto no sólo fue informativo, sino también experiencial, representando un momento de intercambio, que pudo explotar el efecto de "elección de grupo" sin el sentido de la obligación, trayendo a varios niños que normalmente no comen fruta para comerla. Además, incluso 
en el caso de los niños que suelen comer fruta, el beneficio se obtuvo aumentando la confianza en sí mismos, aboliendo el sentido de inferioridad que a veces puede acompañar a los niños que comen fruta como tentempié.

En general, tanto en el desayuno como en la merienda que se come en la escuela, en muchos casos la fruta ha sustituido a las meriendas habituales, como los sándwiches con salchichas, chocolates, salchichas, bebidas endulzadas, haciendo que muchos niños descubran la bondad de la fruta.

Si bien el porcentaje de niños que desayunan sigue siendo el mismo (alrededor del 90\%), se han producido cambios a nivel "cualitativo": el consumo de té y bizcochos ha aumentado, mientras que el consumo de galletas ha disminuido enormemente y alrededor del $15 \%$ de los niños han empezado a comer pan y mermelada. Todavía hay un $10 \%$ de niños que no desayunan por la mañana antes de ir a la escuela, y esto nos hace pensar que es necesaria una intervención de otro tipo.

También se ha encontrado un cambio importante en la calidad de la merienda que se consume en la escuela: muchos niños empiezan a consumir fruta, galletas y yogur, a expensas de las meriendas, las galletas y los zumos de fruta. En definitiva, se desprende que casi todos los niños, en la fase final, hacen una merienda adecuada, mientras que en la fase inicial eran sólo un tercio de la muestra.

Incluso el hábito de tomar un bocadillo por la noche después de la cena, después de la cirugía, parece desaparecer, comparado con el 8\% que lo hacía, antes de la cirugía.

Estos cambios muestran que una intervención dirigida, que involucre tanto a las familias como a los niños, sobre la importancia del desayuno y los beneficios de la fruta, puede ser eficaz y provocar cambios en los hábitos alimenticios, al menos a corto plazo. Sería interesante evaluar, y esto representa una limitación de la investigación actual, pero al mismo tiempo un estímulo para futuras investigaciones, el efecto en un tiempo más largo, a través de un estudio longitudinal.

\section{El sedentarismo y la actividad física antes y después de la intervención}

Numerosos estudios han demostrado la estrecha correlación que existe entre las actividades sedentarias y el riesgo de sobrepeso y obesidad (Trojan, Macera, \& Ballard-Barbash, 2001), y el frecuente hábito de no jugar al aire libre 0 hacer deporte estructurado. Hoy en día, Ios niños prefieren las actividades sedentarias, como ver la televisión, escuchar música, jugar a los videojuegos, sentarse frente a una tableta, una computadora 0 un teléfono inteligente, y a menudo asocian estas actividades con la ingesta de alimentos de bajo valor nutritivo y alto contenido calórico (Vader, Walters, Harris \& Hoelscher, 2009).

En este aspecto, así como en los hábitos alimenticios, los resultados de nuestro estudio no son alentadores, pero reflejan las tendencias nacionales e internacionales. Más de la mitad de los niños ya ven la televisión por la mañana antes de ir a la escuela y el porcentaje de niños que la ven por la tarde y también por la noche después de la cena se eleva a casi tres cuartos de la muestra. A estos hábitos hay que añadir que alrededor de un tercio de los niños entrevistados tienen el hábito de jugar a los videojuegos y al ordenador todas las tardes durante más de tres horas, y casi el 20\% de los participantes lo hacen por la noche después de la cena.

Nos pareció importante detectar el mismo comportamiento en diferentes momentos del día, porque en nuestra opinión se trata de dos variables diferentes, es decir, cualitativamente (y no sólo cuantitativamente) comportamientos diferentes: jugar una hora con videojuegos durante la tarde puede representar un comportamiento apropiado, mientras que jugar la misma cantidad de tiempo por la noche después de la cena y antes de irse a dormir representa un comportamiento poco saludable.

Los datos provenientes de los padres nos dan una idea más precisa del tiempo que pasan sus hijos frente al televisor y con los videojuegos, de hecho la mayoría de ellos informaron que su hijo juega una hora al día, alrededor de un cuarto juega dos horas, alrededor de un 5\% durante 3-4 horas y sólo el 3\% restante juega menos de una hora al día. Los padres informan de períodos de tiempo aún más largos cuando se trata de la televisión: alrededor del 18\% pasa entre 3 y 5 horas viendo la televisión, alrededor del 37\% pasa dos horas al día frente a la pantalla, el 43,42\% ve la televisión durante aproximadamente una hora al día y sólo un pequeño 2\% (es decir, sólo 3 de 153 niños) ve menos de una hora al día. Si pensamos en la suma del tiempo dedicado a los videojue- 


\section{LA EDUCACIÓN ALIMENTARIA DE LOS NIÑOS}

gos, la televisión y las computadoras, el resultado nos da la idea de que los niños son muy sedentarios, lo que concuerda con la pequeña cantidad de tiempo que dedican a la actividad física. Aunque practicar un deporte por la tarde es un hábito que afecta a más de la mitad de los niños, jugar al aire libre por la tarde es definitivamente un comportamiento menos común que los videojuegos. A esto añadimos que casi todos los niños van a la escuela acompañados de un coche.

Mediante el cuestionario dirigido a los padres, se investigaron los hábitos de sus hijos durante un período de tiempo más largo, es decir, durante una semana. Específicamente, se preguntó cuántos días a la semana los niños hacen actividad física fuera de la escuela durante al menos una hora y se encontró que una cuarta parte de los niños no hace ninguna actividad física, el 12\% hace sólo un día a la semana, otra cuarta parte de los niños hace actividad física durante dos días a la semana, el porcentaje más alto (32\%) hace actividad física durante 3 días a la semana y un pequeño porcentaje (5\%) hace actividad física durante más de 3 días.

Los padres son conscientes de que sus hijos no realizan actividad física como indica la OMS (al menos una hora al día).

También en este caso encontramos un cambio después de la intervención de sensibilización, de hecho un cambio en la cantidad de tiempo que se pasa en la televisión, que es un factor predictivo de la condición de sobrepeso 0 el riesgo de obesidad de los niños; 21 niños (de 82) abandonaron el hábito de ver la televisión por la mañana antes de ir a la escuela y hasta 59 niños (de 109) no la vieron más por la tarde.

Después de la intervención también se observó un cambio en el uso de los videojuegos y las computadoras; el número de niños que jugaban a los videojuegos disminuyó de 50 niños que los jugaban durante varias horas (antes de la intervención) a sólo 31. Además, de manera muy alentadora, se observó que después de la intervención el hábito de jugar a los videojuegos o a las computadoras por la noche después de la cena casi desapareció, de 30 a 1 niño de un total de 153 en la muestra. La intervención, por lo tanto, tuvo un efecto considerable en este hábito, que ciertamente representa un comportamiento poco saludable.

También se ha encontrado un cambio positivo con respecto al hábito de jugar al aire libre y practicar deporte.

Por consiguiente, es posible decir que una simple intervención de sensibilización ha tenido un efecto importante en diversos comportamientos estrechamente relacionados con la salud física de los niños, y tal vez el éxito esté también vinculado a la decisión de hacer participar a los padres y maestros en la actividad.

\section{CONCLUSIONES}

Aun cuando la investigación-intervención no pretende sacar conclusiones a gran escala, se puede afirmar con certeza que, dados los considerables cambios en el estilo de vida de los niños que participan en el proyecto, que demuestran la eficacia de la intervención, es necesario seguir ejecutando proyectos de prevención y hacerlo de manera aún más generalizada y con la participación de los diversos agentes, como se prevé en la estrategia de la Organización Mundial de la Salud para combatir las enfermedades crónicas Gaining Health, aprobada en septiembre de 2006 por el Comité Regional para Europa (OMS, 2006).

\section{REFERENCIAS BIBLIOGRÁFICAS}

Dasgupta, P., \& Hauspie, R (2013). Perspectives in Human Growth, Development and Maturation. Berlino: Springer Science \& Business Media.

Fabrizio, M. E. (2011). Obesità. Conoscerla per vincerla. Roma: Edizioni Universitarie Romane.

Faliva, C. (2011). Tra normalità e rischio. Manuale di psicologia dello sviluppo e dell'adolescenza. Santarcangelo di Romagna: Maggioli Editore.

Flegal, K.M., Graubard, B.I., Williamson, D.F., \& Gail, M.H. (2007). Cause-specific excess deaths associated with underweigh, overweight, and obesity. Jama, 2007 Nov. 7; 298(17):2028-37.

Gomez-Pinilla, F. (2008). Brai foods: the effects of nutrients on brain function. Nat Rev Neurosci. $2008 \mathrm{Jul}$; 9(7): 568-578. Doi: 10.1038/nrn2421. 
Gremigni, P., \& Letizia, L. (2011). II problema dell'obesità. Manuale per tutti. I professionisti della salute. Santarcangelo di Romagna: Maggioli Editore.

He, M., \& Evans, A. (2007). Les parents sont-ils conscients que leurs enfants souffrent de surpoints ou d'obésité? Canadian Family Physician, 53, 1493-1499.

Hobbs, M., Pearson, N., Foster, P.J., \& Biddle, S.J. (2015). Sedentary behaviour and diet across the lifespan: An updated systematic review. British Journal of Sports Medicine, 49 (18), 1179-1188. doi: 10.1136/bjsports-2014-093754.

Huang, J.S., Becerra, K., Oda, T., Walker, E., Xu, R., Donohue, M., Chen, I., Curbelo, V., \& Breslow, A. (2007). Parental ability to discriminate the weight status of children: results of a survey. Pediatrics, 120, 112-119.

Ng, M., Fleming, T. Robinson, M., Thomson, B., Graetz, N., \& Margono, C. (2013). Global, regional, and national prvalence of overweight and obesity in children and adults during 1980-2013: a systematic analysis for the Global Burden of Disease Study 2013. The Lancet, volume 384, issue 9945, pp. 776-781.

OMS (2019). Carta Europea sull'azione di contrasto all'obesità. Disponibile da: http://focus.formez.it/sites/all/files/carta_obesit_istanbul.pdf.

Rhee, K.E., De Lago, C.W., Arscott-Mills, T., Mehta, S.D., \& Davis, R.K. (2005). Factors associated with parental readiness to make changes for overweight children. Pediatrics, 116, pp. 94-101.

Ritchie, H. \& Roser, M. (2019). Obesity. Disponibile online da: https://ourworldindata.org/obesity.

Rotella, C.M. (1980). L'obesità. Manuale per la diagnosi e la terapia. Firenze: SEE.

Troiano, R.P., Macera, C.A., \& Ballard-Barbash, R. (2001). Be physically active each day. How can we know? The Journal of Nutrition, 131, pp. 451-460.

Vader, A.M., Walters, S.T., Harris, T.R., \& Hoelscher, D.M. (2009). Television viewing and snacking behaviors of fourth- and eighth-grade schoolchildren in Texas. Preventing Chronic Disease, 6: A88.

Wang, G., \& Speakman, J.R. (2016). Analysis of Positive Selection at Single Nucleotide Polymorphism Associated with Body Mass Index Does Not Support the "Thrifty Gene" Hypotesis. Cell Metalab. 2016 oct 11; 24 (4):531-541. doi: 10.1016/j.cmet.2016.08.014.

WHO (2006). World Health Organization regional Office for Europe. Gaining Health - The European Strategy for the Prevention and Control of Noncommunicable Diseases. Copenhagen: WHO; 2006.

WHO, (2018). Obesity and overweight. Disponibile da: https://www.who.int/en/news-room/factsheets/detail/obesity-and-overweight.

\section{NOTAS}

1 El proyecto "OKkio alla salute" es un sistema de supervisión bienal sobre el peso, hábitos alimentarios y estilo de vida de los niños de escuelas primarias, coordinado por el Instituto Superior de la Sanidad (ISS). 
